\title{
Correlates of foot pain severity in adults with hallux valgus: a cross-sectional study
}

Sheree E Hurn ${ }^{1,2^{*}}$, Bill T Vicenzino ${ }^{3}$ and Michelle D Smith ${ }^{3}$

\begin{abstract}
Background: Hallux valgus (HV) is highly prevalent and associated with progressive first metatarsophalangeal joint subluxation and osteoarthritis. The link between structural HV deformity and foot pain is unclear. This study investigated possible explanatory factors surrounding foot pain in $\mathrm{HV}$, including radiographic $\mathrm{HV}$ angle and signs of joint degeneration.

Methods: Participants were 60 adults (53 female) with HV aged 20 to 75 years. Participant demographics and a range of radiographic, clinical and functional measures were considered potential correlates of foot pain. Self-reported foot pain (visual analogue scales and a dichotomous definition) was considered the dependent variable. Multivariate modelling was used to determine which characteristics and measures explained pain, with univariate analyses first used to screen potential variables.
\end{abstract}

Results: Approximately 20 to 30\% of the variance in foot pain associated with HV could be explained by patient characteristics such as poorer general health status, lower educational attainment and increased occupational physical activity levels, in combination with some dynamic physical characteristics such as hallux plantarflexion weakness and reduced force-time integral under the second metatarsal during gait. Neither increasing lateral deviation of the hallux ( $\mathrm{HV}$ angle) nor presence of first metatarsophalangeal joint osteoarthritis was associated with foot pain.

Conclusions: This study shows that passive structural factors, including HV angle, do not appear to be significant correlates of foot pain intensity in HV. Our data demonstrate the importance of considering patient characteristics such as general health and physical activity levels when assessing foot pain associated with HV.

\section{Background}

Hallux valgus (HV) is a highly prevalent forefoot deformity [1], presenting with lateral deviation of the first toe and progressive subluxation of the first metatarsophalangeal (MTP) joint. HV is often associated with first MTP joint osteoarthritis (OA) [2] and has been linked to impaired physical function $[3,4]$ and poorer general health and quality of life [3-5]. However, there have been contradictory reports regarding the link between foot pain and HV [4,6-10].

Pain associated with HV is often attributed to local mechanical stimuli [11] or degenerative processes within

\footnotetext{
*Correspondence: s.nix@qut.edu.au

'Queensland University of Technology, School of Clinical Sciences, Kelvin Grove, QLD 4059, Australia

${ }^{2}$ Queensland University of Technology, Institute of Health and Biomedical Innovation, Kelvin Grove, QLD 4059, Australia

Full list of author information is available at the end of the article
}

the first MTP joint-sesamoid complex [2]. It could therefore be hypothesised that more severe deformity may be associated with more severe pain, and one previous study has found a positive association between self-reported $\mathrm{HV}$ severity and increasing pain levels [4], although being a population survey radiographic measures were not used. Interestingly, radiographic studies of other lower limb conditions have found no association between pain levels and radiographic measures of knee OA [12] or first MTP joint OA [13]. No previous study has comprehensively investigated the range of foot and ankle characteristics that might explain foot pain symptoms in HV, including plantar pressures [14,15], plantar hyperkeratosis [16], muscle weakness [3] and footwear [17].

In addition to foot and ankle factors, pain severity may be influenced by demographic, cognitive and lifestyle factors, with previous studies showing foot pain to be worse in females, older people, those with higher 
body mass index (BMI) and lower educational attainment $[3,7,13,18-20]$. A study of pain symptoms in HV should therefore investigate local factors as well as patient characteristics.

This cross-sectional study examined potential explanatory factors surrounding foot pain in $\mathrm{HV}$, including radiographic $\mathrm{HV}$ angle and signs of $\mathrm{OA}$, as well as foot and ankle factors and participant characteristics. Differences between participants with and without disabling foot pain were also investigated.

\section{Methods}

\section{Participants}

Participants with HV (defined as a radiographic HV angle greater than 15 degrees) were recruited through community advertisements. Potentially eligible volunteers aged 20 years and older were screened for the following exclusion criteria: history of foot or ankle surgery or fractures, inflammatory disease, neurological conditions and history of falls. Because radiographs were required for this study, pregnant or breastfeeding women were also excluded. In order to exclude cases with concomitant hallux limitus, HV participants were required to have a minimum of 50 degrees passive dorsiflexion at the first MTP joint [21]. Ethical approval was gained from The University of Queensland Medical Research Ethics Committee, and all participants gave written informed consent prior to involvement in this study.

\section{Measurement procedure \\ Foot pain}

Foot pain intensity was measured on a $100 \mathrm{~mm}$ visual analogue scale (VAS), with $0 \mathrm{~mm}$ described as "no pain" and $100 \mathrm{~mm}$ described as "worst pain ever." Participants were asked to indicate their worst level of foot pain and average level of foot pain experienced over the past four weeks. The examiner marked the pain location(s) on a diagram. To further describe foot pain as it relates to functional disability, participants completed the Manchester Foot Pain and Disability Index [22]. If one of the 10 functional limitation items was reported to occur on "most/ every day", participants were considered to have "disabling foot pain" [23].

\section{Participant characteristics}

Demographic data including sex, age, ethnicity, and level of education were obtained via questionnaire. Height and weight were recorded and BMI was calculated. General health was assessed using the SF-36v2 ${ }^{\circ}$ Health Survey general health subscale (score range $0-100$, with higher scores indicating better general health) [24]. Habitual physical activity levels were assessed using the Baecke questionnaire to calculate a work, sport, and leisure index [25].

\section{Assessment of HV}

Bilateral weight bearing dorsoplantar radiographs were obtained for all participants using a standardised procedure (tube to film distance $100 \mathrm{~cm}$, angled 15 degrees from vertical). Alignment between the first metatarsal and proximal phalanx, or HV angle, was measured using computer software developed for telemedical applications [26]. Dorsoplantar radiographs were then examined for presence of OA at the first MTP joint [27,28]. Severity of HV deformity was also graded clinically using the Manchester Scale $[29,30]$ and both feet were examined for the presence of forefoot calluses, recorded by the examiner as "present" or "absent."

\section{Pressure-pain threshold}

Pressure-pain threshold was measured at the medial and plantar aspects of the first MTP joint to obtain a quantifiable measurement of mechanical hyperalgesia. A digital pressure algometer (Somedic AB, Farsta, Sweden) was used to measure pressure, which was applied at a rate of $40 \mathrm{kPa} / \mathrm{s}$ via a rubber-tipped probe $\left(\right.$ area $1 \mathrm{~cm}^{2}$ ). Participants were asked to indicate when the sensation of pressure changed to onset of pain. An average of three measurements from each site was used for analysis.

\section{Foot posture and joint mobility}

Foot posture was assessed using the six-item Foot Posture Index [31-33], which provides a score ranging from -12 to +12 , indicating a supinated (negative) or pronated (positive) foot type. Dorsal arch height (DAH) and midfoot width (MFW) were measured in weight bearing and nonweight bearing using a protocol previously described [34]. The difference between weight bearing and non-weight bearing DAH and MFW provides an indication of foot mobility, and foot mobility magnitude (FMM) is a composite measure representing change in both $\mathrm{DAH}$ and MFW. Generalised joint hypermobility was assessed using the nine-point Beighton scale [35]. The weight bearing lunge test was used to assess dorsiflexion range of motion (ROM) at the ankle joint [36] and passive dorsiflexion ROM at the first MTP joint was assessed in non-weight bearing using a small goniometer [21].

\section{Hallux plantarflexion and abduction strength}

Hallux plantarflexion and abduction strength were evaluated using $50 \mathrm{~kg}$ load cells (GK 2126-50, Gedge Systems, Melbourne, Australia) mounted in a custom-built frame [37]. Participants performed three maximum isometric voluntary contractions in each direction while seated, and the maximum force achieved over the three trials was used for analysis. For hallux abduction, the load cell sensor was positioned so that it was just touching the medial aspect of the hallux in each participant's resting position. Participants were then instructed to "spread their toes 
apart sideways." All participants were able to produce some movement of the hallux given this instruction, although the amount of control to abduct the hallux towards the sensor varied greatly between participants, especially those with moderate to severe HV deformity. The paper grip test was used as a clinical measure of hallux plantarflexion strength $[38,39]$. Participants completed three trials of three seconds, and a pass was recorded if the individual could hold the paper under the hallux against resistance for all trials.

\section{Plantar pressures}

The Pedar- $\mathrm{X}^{\oplus}$ system (Novel ${ }_{\text {gmbh }}$, Munich, Germany) was used to evaluate in-shoe plantar pressures. Insoles were fitted in sports shoes or walking shoes provided by the participants who completed five walking trials at a selfselected comfortable speed along a 10 metre walkway. Analysis was based on data from an average of 23 steps over the 5 trials. Five forefoot regions were identified using a relative mask based on prior work by Putti et al. [40]: hallux, lesser toes, first metatarsal, second metatarsal, third to fifth metatarsals. In each region four different parameters were evaluated: peak pressure $(\mathrm{kPa})$, pressure-time integral $(\mathrm{kPa} * \mathrm{~s})$, maximum force $(\% \mathrm{BW})$ and force-time integral (\%BW*s).

\section{Footwear assessment}

In addition to the shoes worn for Pedar ${ }^{\circledR}$ analysis, participants were asked to bring the two pairs of shoes they wore most frequently. Shoes worn to the examination session were assessed for relative heel height using methods previously described [41]. Forefoot ball width was determined using digital callipers to measure the horizontal distance across the widest point of the MTP joints. The callipers were then used to measure the shoe across the widest point of the forefoot, and relative ball width was calculated as the difference between these two measures. Finally, participants were asked: "Have you ever worn shoes with heels two inches high or greater?" (Yes/No) and "How often do you currently wear this style of shoe?" (Never, Seldom, Sometimes, Often, Always) [41].

All measurements were obtained by the principal examiner. Intrarater reliability for several of these measurement methods has been discussed previously [37]: very good reliability (ICCs $\geq 0.90$ ) was found for footwear assessment and radiographic HV angle measurement, good reliability (ICCs $\geq 0.75$ ) was found for pressure-pain threshold at the first MTP joint and hallux plantarflexion strength, while hallux abduction strength measures were moderately reliable $(\mathrm{ICC}=0.73)$ [42].

\section{Sample size determination}

Sample size calculations based on preliminary data analysis indicated that a sample size of 54 would have $90 \%$ power to detect a significant effect of HV angle when added to a model with five other predictor variables (alpha level $=0.05 ; R^{2}$ change $=0.05$ ). With a $10 \%$ allowance for missing data, a sample size of 60 was obtained for this study.

\section{Statistical analysis}

For variables measured bilaterally, only the right or left foot was chosen for analysis [43] based on the greater radiographic $\mathrm{HV}$ angle (28 right feet and 32 left feet). Data were screened for normality of distribution, and continuous variables showing a skewed distribution were transformed prior to analysis using an appropriate transformation (log, square or inverse). Means, standard deviations (SD) and frequencies were calculated for the entire sample and for those with and without disabling foot pain [23]. Differences between participants with and without disabling foot pain were investigated using independent t-tests for continuous variables and Chi-squared tests for categorical variables. The level of significance for these tests was $p<0.05$.

A two-step approach was used to examine multivariate correlates of foot pain intensity (worst and average foot pain VAS). First, univariate analyses were performed to investigate potential associations between average or worst foot pain VAS and each independent variable. Pearson's or Spearman's correlations were used for continuous variables, and independent $\mathrm{t}$-tests were used for dichotomous variables. Variables that were categorical were analysed using a one-way analysis of variance with the categorical variable as the grouping variable. Potential predictor variables included the following continuous variables: age, BMI, activity level (work, sport and leisure indices), general health (SF-36v2 $2^{\circ}$, HV angle, pressure-pain threshold, ankle lunge test, first MTP joint dorsiflexion, difference between weight bearing and non-weight bearing DAH and MFW, FMM, plantar pressure parameters (peak pressure, pressure-time integral, maximum force, force-time integral), and footwear measurements (relative heel height, relative ball width). Dichotomous and categorical variables included: sex, ethnicity, education, history and frequency of wearing high heeled shoes, Manchester Scale, presence of forefoot callous, first MTP joint OA, and the paper grip test. The Foot Posture Index and Beighton joint hypermobility score were considered ordinal scales and therefore Spearman's rho was used to investigate univariate associations with pain VAS, and the Wilcoxon rank-sum test was used to examine differences between those with and without disabling foot pain. Second, variables found to be potentially associated with worst or average foot pain VAS (significance level for screening, $p<0.1$ ) were entered into a series of multiple linear regression models on the basis of the strength of their univariate associations. Change in the amount of variance $\left(R^{2}\right)$, standardised beta weights 
Table 1 Participant characteristics and foot and ankle characteristics in those with and without disabling foot pain Total $(n=60) \quad$ Disabling foot pain $(n=16) \quad$ No disabling foot pain $(n=44)$ MD $(95 \% \mathrm{Cl})$ or $p$ value

\section{Participant characteristics}

Sex (n female)

Age (years)

BMI $\left(\mathrm{kg} / \mathrm{cm}^{2}\right)$

Ethnicity (n Caucasian)

Education $(n)^{a}$

High school
Trade/diploma/certificate

Degree

Postgraduate

General health score (SF-36v2)

Physical activity (score range 1 to 5 )

$$
\begin{aligned}
& \text { Work index } \\
& \text { Sport index } \\
& \text { Leisure index }
\end{aligned}
$$

Footwear examination ${ }^{b}$

Relative heel height $>25 \mathrm{~mm}(\mathrm{n}(\%))$
Relative ball width $(\mathrm{mm})$

History of wearing high heels $(\mathrm{n}(\%))^{\mathrm{a}}$

Frequency of wearing high heels $(n)^{a}$

Never
Seldom

Sometimes

Often

Always

\section{Foot and ankle characteristics}

Radiographic hallux valgus angle $\left(^{\circ}\right)$

Manchester Scale (n)

Grade 0 (none)

Grade 1 (mild)

Grade 2 (moderate)

Grade 3 (severe)

First MTP joint dorsiflexion $\left(^{\circ}\right)$

Presence first MTP joint OA (n (\%))

Presence forefoot callous (n (\%))

Foot Posture Index (median (min, max))

Dorsal arch height difference $(\mathrm{mm})$

Midfoot width difference $(\mathrm{mm})$

Foot Mobility Magnitude (mm)

Ankle lunge test $(\mathrm{cm})$

Beighton scale (median (min, max))

$\begin{array}{cc}53 & 15 \\ 51.5 \pm 15.1 & 56.6 \pm 12.0 \\ 25.1 \pm 4.3 & 25.4 \pm 3.1\end{array}$

50

12

13

15

17

14

$78.7 \pm 14.9$

$2.63 \pm 0.35$

$2.68 \pm 0.90$

$2.86 \pm 0.61$

$8(13.8 \%)$

$4.7 \pm 4.6$

$29(49.2 \%)$

21
21
12
3
2

$29.5 \pm 8.0$

$31.1 \pm 7.8$

0

1

7

8

$83.8 \pm 11.0$

$11(18.3 \%)$

$84.1 \pm 9.7$

44 (73.3\%)

$7(-3,11)$

$12.5 \pm 4.3$

$9.0 \pm 3.5$

$15.8 \pm 4.3$

$11.5 \pm 3.1$

$1(0,9)$
38

$49.7 \pm 15.7$

$25.0 \pm 4.6$

38

9

9

15

10

$81.0 \pm 14.8$

$2.58 \pm 0.37$

$2.75 \pm 0.91$

$2.86 \pm 0.56$

$6(14.0 \%)$

$4.6 \pm 5.0$

$21(47.7 \%)$

17

13

9

3

2

$28.9 \pm 8.0$

8

19

16

$83.6 \pm 11.5$

8 (18.2\%)

$31(70.5 \%)$

$7(-3,11)$

$11.8 \pm 3.7$

$9.0 \pm 3.2$

$15.1 \pm 3.8$

$11.5 \pm 3.3$

$1(0,6)$
$2.2(-2.5$ to 6.8$)$

$p=0.57$

$p=0.43$

6.9 (-1.8 to 15.6$)$

0.3 (-2.2 to 2.8$)$

$p=0.55$

$p=0.34$

$-8.8(-17.3 \text { to }-0.29)^{*}$

$0.17(-0.04$ to 0.37$)$

$-0.26(-0.79$ to 0.27$)$

$-0.01(-0.37$ to 0.35$)$

$$
p=0.95
$$

0.5 (-2.4 to 3.4$)$

$p=0.71$

$p=0.42$

$$
p=0.57
$$

0.45 (-6.0 to 6.9)

$p=0.96$

$p=0.40$

$p=0.93^{c}$

$2.9(0.47 \text { to } 5.3)^{*}$

$0.27(-1.8$ to 2.3$)$

$2.7(0.22 \text { to } 5.2)^{*}$

-0.01 (-1.8 to 1.8$)$

$$
p=0.42^{c}
$$


Table 1 Participant characteristics and foot and ankle characteristics in those with and without disabling foot pain (Continued)

\begin{tabular}{|c|c|c|c|c|}
\hline Paper grip test (n pass (\%)) & $13(21.7 \%)$ & $1(6.3 \%)$ & $12(27.3 \%)$ & $p=0.08$ \\
\hline \multicolumn{5}{|l|}{ Hallux muscle strength (N) } \\
\hline Plantarflexion & $60.7 \pm 28.3$ & $51.6 \pm 30.9$ & $64.0 \pm 26.8$ & $-12.4(-28.8$ to 3.9$)$ \\
\hline Abduction & $8.9 \pm 6.6$ & $7.5 \pm 6.4$ & $9.4 \pm 6.7$ & $-2.0(-5.8$ to 1.9$)$ \\
\hline \multicolumn{5}{|l|}{ Pressure-pain threshold (kPa) } \\
\hline Medial first MTP joint & $591.5 \pm 231.4$ & $552.5 \pm 219.9$ & $605.7 \pm 236.3$ & $-53.2(-188.9$ to 82.5$)$ \\
\hline Plantar first MTP joint & $442.5 \pm 193.1$ & $428.9 \pm 230.8$ & $447.5 \pm 180.3$ & $-18.5(-132.2$ to 95.2$)$ \\
\hline
\end{tabular}

and $p$-values were examined to determine if variables made a significant independent contribution to the model, and independent variables were retained in the model if $p \leq 0.05$. Finally, radiographic $\mathrm{HV}$ angle was forced into the model to investigate any possible contribution of $\mathrm{HV}$ severity to foot pain. Regression models were checked for multicollinearity and normality of residuals. All statistical analyses were conducted using Stata version 10 (StataCorp LP, College Station, TX).

\section{Results}

Participants were 53 women and 7 men with HV, aged 20 to 75 years. HV angles of participants ranged from 15.5 to 54.4 degrees. All participant characteristics (frequencies, means and SDs) are presented in Table 1.

\section{Foot pain locations}

Qualitative examination of foot pain locations revealed that the most common site of reported pain was the first MTP joint $(n=36,60 \%)$, while pain in the lesser MTP joints $(n=16,27 \%)$, hallux $(n=7,12 \%)$ and lesser toes $(\mathrm{n}=4,7 \%)$ was also reported. Midfoot pain $(\mathrm{n}=16,27 \%)$ and heel pain $(n=7,12 \%)$ were reported by some participants. Thirty-two individuals reported pain at one site, 18 reported pain at two sites, while six individuals reported pain at three different sites on the foot diagram.

\section{Associations with disabling foot pain}

Sixteen participants were defined as having disabling foot pain [23]. Participant characteristics and foot and ankle characteristics for those with and without disabling foot pain are presented in Table 1. Those with disabling foot pain reported significantly poorer general health (mean difference (MD) -8.8, 95\% CI: -17.3 to -0.29 ). Both DAH difference (MD $2.9 \mathrm{~mm}, \mathrm{CI}: 0.47$ to 5.3 ) and FMM (MD $2.7 \mathrm{~mm}, \mathrm{CI}: 0.22$ to 5.2 ) were significantly increased in those with disabling foot pain. Those with disabling foot pain were slightly older than those without disabling foot pain (MD 6.9 years, CI: -1.8 to 15.6 ) but this difference was not statistically significant. No other participant characteristics, foot and ankle characteristics or footwear factors showed significant differences between those with and without disabling foot pain. Notably, neither radiographic $\mathrm{HV}$ angle nor the presence of first MTP joint OA was found to be significantly associated with disabling foot pain.

\section{Univariate associations with foot pain VAS}

Table 2 shows univariate associations between foot pain VAS, participant characteristics and foot and ankle characteristics. Lower educational attainment was associated with higher average foot pain VAS ( $\mathrm{p}=0.02)$, while poorer general health scores and higher work activity was associated with higher average and worst reported pain $(\mathrm{p} \leq 0.05)$. Participants who wore shoes with a heel height $>25 \mathrm{~mm}$ to the examination session reported higher worst foot pain $(\mathrm{p}=0.06)$, and those who failed the paper grip test reported higher average and worst foot pain $(\mathrm{p}<0.05)$. An increased arch height difference between non weightbearing and weight-bearing was associated with higher average and worst foot pain $(\mathrm{p}<0.1)$, but interestingly lower Beighton scores indicating less generalised joint hypermobility were associated with higher average pain $(\mathrm{p}=$ 0.09). Correlations between foot pain and plantar pressure and force parameters are displayed in Table 3. The only in-shoe plantar pressure parameters that showed significant correlations with increasing foot pain were reduced force-time integrals under the first and second metatarsal heads $(\mathrm{p}<0.1)$.

\section{Multiple regression analysis}

Multiple regression modelling (Table 4) showed that general health status, educational attainment, work activity index and force-time integral under the second 
Table 2 Univariate associations between participant characteristics, foot and ankle characteristics and foot pain

\begin{tabular}{|c|c|c|c|c|}
\hline & \multicolumn{2}{|c|}{ Average pain VAS } & \multicolumn{2}{|c|}{ Worst pain VAS } \\
\hline & Univariate association & $p$ value & Univariate association & $p$ value \\
\hline \multicolumn{5}{|l|}{ Participant characteristics } \\
\hline Gender & $\mathrm{MD}=1.3 \mathrm{~mm}$ & 0.85 & $\mathrm{MD}=6.9 \mathrm{~mm}$ & 0.56 \\
\hline Age & Pearson's $r=0.15$ & 0.25 & Pearson's $r=0.06$ & 0.66 \\
\hline$B M l^{\mathrm{a}}$ & Pearson's $r=0.07$ & 0.61 & Pearson's $r=0.04$ & 0.77 \\
\hline Ethnicity & ANOVA $F=0.12$ & 0.88 & ANOVA $F=0.16$ & 0.85 \\
\hline Education & ANOVA F $=3.46$ & $0.02^{*}$ & ANOVA $F=1.50$ & 0.22 \\
\hline General health score (SF-36v2) & Pearson's $r=-0.32$ & $0.01^{*}$ & Pearson's $r=-0.26$ & $0.05^{*}$ \\
\hline \multicolumn{5}{|l|}{ Physical activity level } \\
\hline Work index & Pearson's $r=0.32$ & $0.02^{*}$ & Pearson's $r=0.34$ & $0.009^{*}$ \\
\hline Sport index ${ }^{\mathrm{a}}$ & Pearson's $r=0.05$ & 0.73 & Pearson's $r=0.01$ & 0.92 \\
\hline Leisure index & Pearson's $r=0.00$ & 0.98 & Pearson's $r=-0.03$ & 0.81 \\
\hline \multicolumn{5}{|l|}{ Footwear worn to examination } \\
\hline Relative heel height $>25 \mathrm{~mm}$ & $\mathrm{MD}=5.1 \mathrm{~mm}$ & 0.46 & $\mathrm{MD}=20.6 \mathrm{~mm}$ & $0.06^{*}$ \\
\hline Relative ball width & Pearson's $r=-0.01$ & 0.97 & Pearson's $r=-0.10$ & 0.46 \\
\hline History of wearing high heels & $\mathrm{MD}=6.3 \mathrm{~mm}$ & 0.17 & $\mathrm{MD}=5.3 \mathrm{~mm}$ & 0.49 \\
\hline Frequency of wearing high heels & ANOVA F $=1.07$ & 0.38 & ANOVA F $=0.40$ & 0.81 \\
\hline \multicolumn{5}{|l|}{ Foot and ankle characteristics } \\
\hline Radiographic hallux valgus angle & Pearson's $r=-0.02$ & 0.88 & Pearson's $r=0.03$ & 0.80 \\
\hline Manchester Scale (grade 0 to 3) & ANOVA $F=1.05$ & 0.38 & ANOVA $F=1.17$ & 0.33 \\
\hline Presence first MTP joint OA & $\mathrm{MD}=6.1 \mathrm{~mm}$ & 0.31 & $\mathrm{MD}=5.9 \mathrm{~mm}$ & 0.55 \\
\hline Presence forefoot callous & $\mathrm{MD}=4.1 \mathrm{~mm}$ & 0.44 & $\mathrm{MD}=3.7 \mathrm{~mm}$ & 0.67 \\
\hline Foot Posture Index (score -12 to +12 ) & Spearman's rho $=0.06$ & 0.64 & Spearman's rho $=-0.04$ & 0.73 \\
\hline Dorsal arch height difference & Pearson's $r=0.26$ & $0.05^{*}$ & Pearson's $r=0.23$ & $0.07^{*}$ \\
\hline Midfoot width difference & Pearson's $r=-0.12$ & 0.41 & Pearson's $r=-0.11$ & 0.42 \\
\hline Foot Mobility Magnitude & Pearson's $r=0.16$ & 0.22 & Pearson's $r=0.14$ & 0.29 \\
\hline First MTP joint dorsiflexion & Pearson's $r=-0.15$ & 0.24 & Pearson's $r=-0.01$ & 0.93 \\
\hline Ankle lunge test & Pearson's $r=0.07$ & 0.59 & Pearson's $r=0.05$ & 0.73 \\
\hline Beighton score (range 0 - 9) & Spearman's rho $=-0.22$ & $0.09^{*}$ & Spearman's rho $=-0.09$ & 0.50 \\
\hline Paper grip test (pass/fail) & $\mathrm{MD}=12.7 \mathrm{~mm}$ & $0.02^{*}$ & $\mathrm{MD}=26.4 \mathrm{~mm}$ & $0.003^{*}$ \\
\hline \multicolumn{5}{|l|}{ Hallux muscle strength } \\
\hline Plantarflexion & Pearson's $r=-0.10$ & 0.43 & Pearson's $r=-0.10$ & 0.44 \\
\hline Abduction $^{a}$ & Pearson's $r=-0.08$ & 0.55 & Pearson's $r=-0.01$ & 0.92 \\
\hline \multicolumn{5}{|l|}{ Pressure-pain threshold } \\
\hline Medial first MTP joint & Pearson's $r=-0.15$ & 0.26 & Pearson's $r=-0.14$ & 0.30 \\
\hline Plantar first MTP joint & Pearson's $r=-0.12$ & 0.38 & Pearson' $r=-0.19$ & 0.14 \\
\hline
\end{tabular}

VAS: visual analogue scale; MD: mean difference; SF-36v2: Short Form 36 Health Survey, version 2.

* Statistically significant at alpha $p<0.1$.

${ }^{\text {a }}$ Transformed (log, inverse or square).

metatarsal head explained 33\% of the variance in average foot pain intensity over the past four weeks. As seen by the beta weights in Table 4, poorer general health, lower educational attainment, a higher work activity index, and lower second metatarsal force-time integrals were associated with increased average foot pain. With worst foot pain VAS as the dependent variable, the two significant contributors to the model were the paper grip test and work activity index, together explaining $20 \%$ of the variance in worst foot pain (Table 5). A failed paper grip test and higher work activity index were associated with increased worst foot pain. No other potential predictor 
Table 3 Correlations between worst and average foot pain VAS and plantar pressure and force parameters

\begin{tabular}{|c|c|c|c|c|c|c|c|c|c|c|}
\hline & \multicolumn{5}{|c|}{ Peak pressure (kPa) } & \multicolumn{5}{|c|}{ Pressure-time integral (kPa*s) } \\
\hline & $M 1^{\mathrm{a}}$ & $M 2^{a}$ & $M 3-5^{a}$ & Hallux & LT & $M 1^{a}$ & $M 2^{\mathrm{a}}$ & $M 3-5^{a}$ & Hallux & $\mathrm{LT}^{\mathrm{a}}$ \\
\hline Average pain & -0.08 & 0.02 & 0.07 & 0.14 & 0.07 & -0.12 & -0.07 & 0.01 & 0.03 & -0.02 \\
\hline \multirow[t]{3}{*}{ Worst pain } & -0.01 & -0.02 & 0.01 & 0.09 & 0.03 & 0.03 & -0.01 & 0.05 & 0.06 & -0.02 \\
\hline & \multicolumn{5}{|c|}{ Maximum force (\% BW) } & \multicolumn{5}{|c|}{ Force-time integral $(\% \mathrm{BW} * \mathbf{s})$} \\
\hline & M1 & M2 & M3-5 & Hallux & LT & M1 & M2 & M3-5 & Hallux & LT \\
\hline Average pain & -0.18 & -0.20 & -0.04 & 0.00 & 0.06 & $-0.23^{*}$ & $-0.23^{*}$ & -0.14 & -0.05 & -0.00 \\
\hline Worst pain & 0.08 & -0.11 & -0.01 & -0.06 & 0.01 & 0.03 & -0.10 & -0.08 & -0.08 & -0.04 \\
\hline
\end{tabular}

VAS: visual analogue scale; M: metatarsal head; LT: lesser toes;\% BW: percentage body weight.

*Statistically significant at alpha $p<0.1$.

aLog transformed.

variables were retained in the multiple regression models as they did not make a significant contribution $(p>0.05)$ (Tables 4 and 5). HV angle was a poor predictor of pain intensity when forced into the final model for average foot pain ( $0 \%$ change in $\mathrm{R}^{2}$, beta $\left.0.007, p=0.96\right)$ and worst foot pain (change in $\mathrm{R}^{2} 0.4 \%$, beta $0.063, p=0.61$ ).

\section{Discussion}

The intensity of foot pain experienced by individuals with $\mathrm{HV}$ is not determined by angular deformity or other passive structural factors, but rather is influenced by patient characteristics such as poorer general health status and increased occupational physical activity levels. In addition to patient factors, some dynamic foot and ankle characteristics were significantly associated with increasing foot pain in this study, such as hallux plantarflexion weakness determined by failure of the paper grip test, and reduced forcetime integral under the second metatarsal during gait. In combination these factors explained approximately 20 to $30 \%$ of the variance in foot pain associated with HV. Clearly, a large proportion (approximately 70 to $80 \%$ ) of the variance remains unexplained by the comprehensive set of factors investigated in this study, and further research is warranted to explore the complexity of foot pain associated with HV.

Our investigation included several structural foot and ankle characteristics frequently assessed by clinicians, including HV angle, radiographic signs of first MTP joint OA, forefoot callous, foot posture and passive joint ROM. Interestingly, participants with first MTP joint OA $(n=$ 11) were not more likely to report disabling foot pain (Table 1) or greater foot pain intensity (Table 2). This finding is consistent with previous studies [13,28], although foot pain is often attributed to degenerative arthritic processes [17]. While forefoot callous is another factor often considered to be correlated with foot pain, both our study and a study by Spink et al. [16] show no association between the presence of plantar hyperkeratotic lesions and foot pain. Furthermore, footwear factors were not significantly associated with disabling foot pain in our study. Having a relative heel height of greater than $25 \mathrm{~mm}$ was potentially associated with worst pain VAS in our

Table 4 Multiple linear regression model ${ }^{\mathrm{a}}$ with average foot pain VAS as the outcome variable

\begin{tabular}{|c|c|c|c|}
\hline Significant predictor variables & Standardised $\beta$ weight & $P$ value & Multiple $\mathrm{R}^{2}$ \\
\hline General health score (SF-36v2) & -0.296 & 0.01 & 0.330 \\
\hline Educational attainment & -0.232 & 0.05 & \\
\hline Work activity index & 0.312 & 0.01 & \\
\hline Force-time integral (M2) & -0.269 & 0.02 & \\
\hline Variables not retained in the model & & & Change in $\mathrm{R}^{2}$ \\
\hline Paper grip test (pass $=0$, fail $=1$ ) & 0.141 & 0.24 & 0.018 \\
\hline Dorsal arch height difference & 0.108 & 0.38 & 0.010 \\
\hline Force-time integral (M1) & -0.106 & 0.47 & 0.007 \\
\hline Beighton Score & -0.144 & 0.22 & 0.020 \\
\hline \multicolumn{4}{|l|}{ Variable forced into the model } \\
\hline Hallux valgus angle & 0.007 & 0.96 & 0.000 \\
\hline
\end{tabular}

VAS: visual analogue scale; SF-36v2: Short Form 36 Health Survey, version 2; M: metatarsal head.

${ }^{a}$ Cases excluded due to missing data $(n=3)$; therefore, analysis based on $n=57$. 
Table 5 Multiple linear regression model ${ }^{a}$ with worst foot pain VAS as the outcome variable

\begin{tabular}{|c|c|c|c|}
\hline Significant predictor variables & Standardised $\beta$ weight & $P$ value & Multiple $\mathrm{R}^{2}$ \\
\hline Paper grip test (pass $=0$, fail $=1$ ) & 0.312 & 0.02 & 0.204 \\
\hline Work activity index & 0.250 & 0.05 & \\
\hline Variables not retained in the model & & & Change in $\mathrm{R}^{2}$ \\
\hline General health score (SF-36v2) & -0.188 & 0.12 & 0.035 \\
\hline Dorsal arch height difference & 0.183 & 0.13 & 0.033 \\
\hline Relative heel height $>25 \mathrm{~mm}$ & 0.193 & 0.13 & 0.035 \\
\hline \multicolumn{4}{|l|}{ Variable forced into the model } \\
\hline Hallux valgus angle & 0.063 & 0.61 & 0.004 \\
\hline
\end{tabular}

univariate analysis $(p=0.06)$, but no footwear variables made a significant contribution to the multivariate regression models.

Dynamic foot and ankle assessments may be more significant than static structural measures in explaining foot pain associated with HV. Failure of the paper grip test helped explain worst foot pain in our multivariate modelling (Table 5). It is plausible that motor weakness surrounding the hallux may occur in response to pain [44]. For example, a previous study by Mickle et al. $(\mathrm{n}=$ 312) [3] reported reduced hallux flexor strength in older adults with disabling foot pain compared to those without disabling foot pain. Further investigation is warranted, as our study sample size may not have provided sufficient power to detect a significant difference in muscle strength between those with and without disabling foot pain (Table 1). In-shoe plantar pressure analysis showed an inverse correlation between average foot pain and force-time integrals under the first and second metatarsal heads (Table 3). Given that the most common site of reported pain was the first MTP joint (60\%), it is plausible that those with more painful feet might walk more cautiously and spend less time loading the medial forefoot when wearing shoes, although other studies have linked higher barefoot plantar pressures to pain in HV [15]. The lack of standardised footwear in our study is an important consideration, as the observed pressures could also reflect characteristics of the footwear. Further studies investigating barefoot versus in-shoe plantar pressures profiles in $\mathrm{HV}$ are warranted.

Our study found that lower educational attainment was associated with higher average pain levels using univariate and multivariate analysis. Cho et al. [7] $(n=563)$ previously reported lower educational attainment to be associated with painful $\mathrm{HV}$ using univariate analysis methods. It is plausible that lower educational attainment could be associated with more physically demanding work, which would be consistent with our finding of a correlation between foot pain and occupational physical activity levels (Table 2). Other studies have reported foot pain to be associated with increasing age [18], female sex $[3,7,18]$ and higher BMI $[3,13,19,20]$, although none of these associations were apparent in our sample of adults with HV.

It is evident that individuals with $\mathrm{HV}$ experience varying levels of foot pain, thus different pain scales and definitions used in previous studies could help explain inconsistent reports. Sixteen out of 60 participants in our study reported disabling foot pain [23], while four individuals reported no foot pain at all, leaving 40 individuals with some degree of mild to moderate foot pain. We chose to use a relatively strict definition (at least one item on the Manchester Foot Pain and Disability Index reported on "most/every day") [23] to differentiate those individuals with disabling foot pain $(\mathrm{n}=16)$ from those without $(\mathrm{n}=$ 44). In addition we used a simple pain VAS to establish pain intensity on a continuous scale. The complex and multidimensional nature of pain, which may be only partially captured by a single-item measure such as the pain VAS, should be acknowledged [45]. Another point to note is that study participants were asked about foot pain, not "big toe pain", which may be more specific to $\mathrm{HV}$. Although the first MTP joint was the most common site of reported pain, some participants reported pain at another site such as the arch or heel, which may not have been directly related to HV. Nonetheless, this is the first study to examine both foot pain intensity and disabling foot pain in HV. Future studies should utilise detailed measures of pain that capture the complexity of this construct in populations with HV.

Several considerations are relevant when interpreting our study findings. The sample size in our investigation was somewhat limited due to the scope of radiographic and clinical measurements obtained, and this may impact on the generalisability of our findings. Our sample size was determined by a priori power calculations and was considered adequate to investigate up to six predictor variables in a multiple regression model. While previous studies have found that painful HV is associated with female sex [7], the number of males in our study was small $(n=7)$, which may explain why our 
study did not find an association between foot pain and female sex. While HV is approximately 2.3 times more prevalent in females compared to males in the general population [1], the gender bias in our study was more pronounced, which may indicate that women were more likely to volunteer to participate. Finally, due to the cross-sectional design of this study, conclusions cannot be drawn regarding which factors may contribute to the development of foot pain, and which factors may develop as a consequence of pain.

\section{Conclusions}

Severity of hallux deviation and radiographic signs of first MTP joint OA are poor predictors of foot pain in HV. Foot pain and disability associated with HV must be evaluated in the context of patient characteristics such as general health status and occupational physical activity levels. Our data suggest that assessment of static foot posture and joint ROMs may be less helpful in guiding management decisions for painful $\mathrm{HV}$, therefore clinical examination of HV should include assessment of dynamic factors such as hallux plantarflexion strength and gait parameters. Due to the high proportion of people with HV who experience foot pain (93\% of our sample), this is an important area for research, yet a large proportion of the variability of pain in $\mathrm{HV}$ remains unexplained. Further research is warranted to understand functional adaptations to foot pain, and to investigate potentially modifiable factors that may contribute to the development of foot pain in HV. Conservative management could then be appropriately targeted to address factors contributing to foot pain and disability in HV.

\section{Competing interests}

The authors declare that they have no competing interests.

\section{Authors' contributions}

SH carried out participant recruitment and data collection, conducted statistical analysis and drafted the manuscript. BV and MS were involved with study conception and design, interpretation of data and critical revision of the manuscript. All authors read and approved the final manuscript.

\section{Acknowledgements}

At the time of conducting the study, Sheree Hurn (nee Nix) was a doctoral candidate at The University of Queensland, School of Health and Rehabilitation Sciences, supported by a Sir Robert Menzies Memorial Scholarship in the Allied Health Sciences. This work was supported by an Australian Podiatry Education and Research Foundation (APERF) grant. The study sponsors had no involvement in the study design, collection, analysis or interpretation of the data.

\section{Author details}

'Queensland University of Technology, School of Clinical Sciences, Kelvin Grove, QLD 4059, Australia. ${ }^{2}$ Queensland University of Technology, Institute of Health and Biomedical Innovation, Kelvin Grove, QLD 4059, Australia. ${ }^{3}$ The University of Queensland, School of Health and Rehabilitation Sciences, Division of Physiotherapy, St Lucia, QLD 4072, Australia.

Received: 22 April 2014 Accepted: 23 June 2014

Published: 28 June 2014

\section{References}

1. Nix S, Smith M, Vicenzino B: Prevalence of hallux valgus in the general population: a systematic review and meta-analysis. J Foot Ankle Res 2010, 3:21.

2. D'Arcangelo P, Landorf KB, Munteanu SE, Zammit GV, Menz HB: Radiographic correlates of hallux valgus severity in older people. J Foot Ankle Res 2010, 3:20.

3. Mickle KJ, Munro BJ, Lord SR, Menz HB, Steele JR: Cross-sectional analysis of foot function, functional ability, and health-related quality of life in older people with disabling foot pain. Arthritis Care Res (Hoboken) 2011, 63:1592-1598

4. Menz HB, Roddy E, Thomas E, Croft PR: Impact of hallux valgus severity on general and foot-specific health-related quality of life. Arthritis Care Res (Hoboken) 2011, 63:396-404.

5. Menz HB, Tiedemann A, Mun-San Kwan M, Plumb K, Lord SR: Foot pain in community-dwelling older people: an evaluation of the Manchester Foot Pain and Disability Index. Rheumatology (Oxford) 2006, 45:863-867.

6. Roddy E, Zhang W, Doherty M: Prevalence and associations of hallux valgus in a primary care population. Arthritis Care Res (Hoboken) 2008, 59:857-862

7. Cho NH, Kim S, Kwon DJ, Kim HA: The prevalence of hallux valgus and its association with foot pain and function in a rural Korean community. J Bone Joint Surg (Br) 2009, 91:494-498.

8. Badlissi F, Dunn JE, Link CL, Keysor JJ, McKinlay JB, Felson DT: Foot musculoskeletal disorders, pain, and foot-related functional limitation in older persons. J Am Geriatr Soc 2005, 53:1029-1033.

9. Nguyen USDT, Hillstrom HJ, Li W, Dufour AB, Kiel DP, Procter-Gray E, Gagnon MM, Hannan MT: Factors associated with hallux valgus in a population-based study of older women and men: the MOBILIZE Boston Study. Osteoarthritis Cartilage 2010, 18:41-46.

10. Garrow AP, Silman AJ, Macfarlane GJ: The Cheshire Foot Pain and Disability Survey: a population survey assessing prevalence and associations. Pain 2004, 110:378-384.

11. Coughlin MJ, Jones CP: Hallux valgus: demographics, etiology, and radiographic assessment. Foot Ank Int 2007, 28:759-777.

12. Barker K, Lamb S, Toye F, Jackson S, Barrington S: Association between radiographic joint space narrowing, function, pain and muscle power in severe osteoarthritis of the knee. Clin Rehabil 2004, 18:793-800.

13. Munteanu SE, Zammit GV, Menz HB: Factors associated with foot pain severity and foot-related disability in individuals with first metatarsophalangeal joint OA. Rheumatology (Oxford) 2012, 51:176-183.

14. Kernozek TW, Elfessi A, Sterriker S: Clinical and biomechanical risk factors of patients diagnosed with hallux valgus. J Am Podiatr Med Assoc 2003, 93:97-103.

15. Wen J, Ding Q, Yu Z, Sun W, Wang Q, Wei K: Adaptive changes of foot pressure in hallux valgus patients. Gait Posture 2012, 36:344-349.

16. Spink MJ, Menz HB, Lord SR: Distribution and correlates of plantar hyperkeratotic lesions in older people. J Foot Ankle Res 2009, 2:8.

17. Riskowski J, Dufour AB, Hannan MT: Arthritis, foot pain and shoe wear: current musculoskeletal research on feet. Curr Opin Rheumatol 2011, 23:148-155.

18. Roddy E, Muller S, Thomas E: Onset and persistence of disabling foot pain in community-dwelling older adults over a 3-year period: a prospective cohort study. J Gerontol A Biol Sci Med Sci 2011, 66:474-480.

19. Tanamas SK, Wluka AE, Berry P, Menz HB, Strauss BJ, Davies-Tuck M, Proietto J, Dixon JB, Jones G, Cicuttini FM: Relationship between obesity and foot pain and its association with fat mass, fat distribution, and muscle mass. Arthritis Care Res (Hoboken) 2012, 64:262-268.

20. Leveille SG, Guralnik JM, Ferrucci L, Hirsch R, Simonsick E, Hochberg MC: Foot pain and disability in older women. Am J Epidemiol 1998, 148:657-665.

21. Hopson MM, MCPoil TG, Cornwall MW: Motion of the first metatarsophalangeal joint: reliability and validity of four measurement techniques. J Am Podiatr Med Assoc 1995, 85:198-204.

22. Garrow AP, Papageorgiou AC, Silman AJ, Thomas E, Jayson MI, Macfarlane GJ: Development and validation of a questionnaire to assess disabling foot pain. Pain 2000, 85:107-113.

23. Roddy E, Muller $S$, Thomas E: Defining disabling foot pain in older adults: further examination of the Manchester Foot Pain and Disability Index. Rheumatology (Oxford) 2009, 48:992-996.

24. Ware JE Jr, Kosinski M, Bjorner JB, Turner-Bowker DM, Gandek B, Maruish ME: User's Manual for the SF-36r2 $2^{\bullet}$ Health Survey. 2nd edition. QualityMetric Incorporated: Lincoln, Rl; 2007. 
25. Baecke JAH, Burema J, Frijters JER: A short questionnaire for the measurement of habitual physical activity in epidemiological studies. Am J Clin Nutr 1982, 36:936-942.

26. Russell T, Jull G, Wootton R: Can the internet be used as a medium to evaluate knee angle? Manual Ther 2003, 8:242-246.

27. Menz HB, Munteanu SE, Landorf KB, Zammit GV, Cicuttini FM: Radiographic classification of osteoarthritis in commonly affected joints of the foot. Osteoarthritis Cartilage 2007, 15:1333-1338.

28. Menz HB, Munteanu SE, Landorf KB, Zammit GV, Cicuttini FM: Radiographic evaluation of foot osteoarthritis: sensitivity of radiographic variables and relationship to symptoms. Osteoarthritis Cartilage 2009, 17:298-303.

29. Garrow AP, Papageorgiou A, Silman AJ, Thomas E, Jayson MI, Macfarlane GJ: The grading of hallux valgus. The Manchester Scale. J Am Podiatr Med Assoc 2001, 91:74-78.

30. Menz HB, Munteanu SE: Radiographic validation of the Manchester scale for the classification of hallux valgus deformity. Rheumatology (Oxford) 2005, 44:1061-1066

31. Redmond AC, Crosbie J, Ouvrier RA: Development and validation of a novel rating system for scoring standing foot posture: The Foot Posture Index. Clin Biomech (Bristol, Avon) 2006, 21:89-98.

32. Cornwall MW, McPoil TG, Lebec M, Vicenzino B, Wilson J: Reliability of the modified Foot Posture Index. J Am Podiatr Med Assoc 2008, 98:7-13.

33. Menz HB, Munteanu SE: Validity of 3 clinical techniques for the measurement of static foot posture in older people. J Orthop Sports Phys Ther 2005, 35:479-486.

34. McPoil TG, Vicenzino B, Cornwall MW, Collins N, Warren M: Reliability and normative values for the foot mobility magnitude: a composite measure of vertical and medial-lateral mobility of the midfoot. J Foot Ankle Res 2009, 2:6.

35. Beighton P, Bird HA, Grahame R: Hypermobility of joints. New York: Springer; 1983.

36. Bennell KL, Talbot RC, Wajswelner H, Technovanich W, Kelly DH, Hall AJ: Intra-rater and inter-rater reliability of a weight-bearing lunge measure of ankle dorsiflexion. Aust J Physiother 1998, 44:175-180.

37. Nix S, Vicenzino BT, Smith MD: Foot pain and functional limitation in healthy adults with hallux valgus: a cross-sectional study. BMC Musculoskelet Disord 2012, 13:197.

38. deWin $M$, Theuvenet $W$, Roche $P$, deBie $R$, vanMameren $H$ : The paper grip test for screening on intrinsic muslce paralysis in the foot of leprosy patients. Int I Lepr Other Mycobact Dis 2002, 70:16-24.

39. Menz HB, Zammit GV, Munteanu SE, Scott G: Plantarflexion strength of the toes: age and gender differences and evaluation of a clinical screening test. Foot Ankle Int 2006, 27:1103-1108.

40. Putti AB, Arnold GP, Cochrane L, Abboud RJ: The pedar ${ }^{\oplus}$ in-shoe system: repeatability and normal pressure values. Gait Posture 2007, 25:401-405.

41. Menz HB, Morris ME: Footwear characteristics and foot problems in older people. Gerontology 2005, 51:346-351.

42. Portney $L G$, Watkins MP: Foundations of clinical research: applications to practice. 3rd edition. Upper Saddle River, N.J: Prentice Hall Health; 2009.

43. Menz HB: Two feet, or one person? Problems associated with statistical analysis of paired data in foot and ankle medicine. Foot 2004, 14:2-5.

44. Butler DS: The Sensitive Nervous System. Adelaide: Noigroup Publications; 2000.

45. Litcher-Kelly L, Martino SA, Broderick JE, Stone AA: A systematic review of measures used to assess chronic musculoskeletal pain in clinical and randomized controlled clinical trials. J Pain 2007, 8:906-913.

doi:10.1186/1757-1146-7-32

Cite this article as: Hurn et al: Correlates of foot pain severity in adults with hallux valgus: a cross-sectional study. Journal of Foot and Ankle Research 2014 7:32.

\section{Submit your next manuscript to BioMed Central and take full advantage of:}

- Convenient online submission

- Thorough peer review

- No space constraints or color figure charges

- Immediate publication on acceptance

- Inclusion in PubMed, CAS, Scopus and Google Scholar

- Research which is freely available for redistribution 